\title{
Picasso à travers quelques périodiques entre 1925 et 1970
}

Recherche présentée par

\section{Maha Ibrahim Salama}

Maître de conférences

Faculté de Jeunes Filles Université Ain shams

\begin{tabular}{|c|c|c|c|}
\hline Nom de l'auteur & Titre de l'article & $\begin{array}{l}\text { Nom de la } \\
\text { Revue }\end{array}$ & Date \\
\hline BRETON, André & $\begin{array}{l}\text { Picasso dans son } \\
\text { élément }\end{array}$ & $\begin{array}{c}\text { Minotaure } \\
n^{\circ} 1\end{array}$ & 1933 \\
\hline BRETON, André & Réponse à une enquête & $\begin{array}{l}\text { Le Surréalisme } \\
\text { et la peinture }\end{array}$ & 1965 \\
\hline ELUARD, Paul & Guernica & $\begin{array}{c}\text { Europe } \\
\mathrm{n}^{\circ} 47\end{array}$ & 1949 \\
\hline ABRAHAM, Pierre & $\begin{array}{l}\text { Note sur l'article } \\
\text { précédent }\end{array}$ & $\begin{array}{c}\text { Europe } \\
\mathrm{n}^{\circ} 47\end{array}$ & 1949 \\
\hline COGNIAT, Raymond & Picasso & $\begin{array}{c}\text { Europe } \\
\mathrm{n}^{\circ} 47\end{array}$ & 1949 \\
\hline GEERINCK, Marianne & $\begin{array}{l}\text { Le Nouvel } \\
\text { Observateur }\end{array}$ & $\begin{array}{c}\text { Histoire de } \\
\text { l'art, le XXème } \\
\text { siècle }\end{array}$ & $1989-1990$ \\
\hline BACHAT, Charles & Picasso et les poètes & $\begin{array}{r}\text { Europe } \\
\mathrm{n}^{0} 48,49 \\
\end{array}$ & 1970 \\
\hline RAYMOND, Jean & Eluard et Picasso & $\begin{array}{c}\text { Europe } \\
\mathrm{n}^{\circ} 48,49 \\
\end{array}$ & 1970 \\
\hline HENRY, Hélène & Max Jacob et Picasso & $\begin{array}{l}\text { Europe } \\
\mathrm{n}^{\circ} 48,49\end{array}$ & 1970 \\
\hline PIERSSENS, Michel & $\begin{array}{l}\text { Apollinaire, Picasso, et } \\
\text { la mort de la poésie }\end{array}$ & $\begin{array}{l}\text { Europe } \\
\mathrm{n}^{\circ} 48,49\end{array}$ & 1970 \\
\hline
\end{tabular}


Non, nous ne mourrons pas tous entiers.

Puisque les choses essentielles d'autres après nous vont les sentir, les toucher, les voir, en parler.

Louis Aragon

Nombreux sont les artistes qui sont considérés immortels pour nous, non pas seulement par des mots, mais par des couleurs. La peinture est donc considérée comme un champ d'influence aussi vaste que la poésie. Elle est comme la poésie parce qu'elle exprime la seconde vie de l'homme.

Avec le début du $\mathrm{XX}^{\mathrm{ème}}$ siècle, se confirment les rapports privilégiés, ténus et féconds entre peintre et poète. Selon René Char, ces deux derniers sont devenus des «alliés substantiels ». Picasso est l'un de ces peintres qui s'est illustré avec talent et art et qui s'est intégré avec les poètes de son siècle.

Il a été le premier à imposer à l'art un certain côté « hors la loi ». C'est pourquoi il a attiré le milieu littéraire et artistique, les uns l'ont admiré, l'ont décrit ou l'ont commenté. Comment l'ont-t-ils perçu ? Dans quelle mesure Picasso a-t-il imposé de nouvelles écoles qui durent peut-être jusqu'à présent? Peut-on dire que c'est sa personne ou plutôt ce sont ses tableaux qui ont attiré l'attention de plusieurs critiques?

Avant de s'installer à Paris, le peintre malaguègne vécut à Barcelone une ambiance favorable à l'éclosion de son génie. Il a participé dans les revues Juventud et Cataluna Artistica à l'émancipation de la langue et de la poésie catalanes. A Paris, Picasso peintre des ballets russes, marié à la très bourgeoise Olga Kokhlova, s'installa rue La Boétie et fréquenta les stations balnéaires à la mode. Il devint riche et surtout de plus en plus célèbre: son génie, son extraordinaire puissance de renouvellement, de remise en question permanente de sa peinture le dispensaient de s'affilier à toute école, le protègaient de la moindre concurrence.

Picasso s'est trouvé intimement lié à la poésie de son temps et en retour «pas un seul poète important qui ne fût inspiré par son œuvre ${ }^{1}$. Nous trouvons 
donc le Picasso de Paul Eluard, le Picasso d'André Salmon, le Picasso de Marcel Béalu ou de René-Guy Cadou.

Trois grands poètes ont été mêlés à la vie du peintre, ils ont vécu avec lui la misère et les moments exaltants des découvertes de son art moderne. Chacun d'eux éclaire un versant déterminé de la peinture de Picasso et chacun a reçu d'elle une empreinte profonde. Apollinaire s'est dépensé pour faire reconnaître et admettre les audaces du jeune peintre. Reverdy, compagnon du «bateau-lavoir », a créé une poésie plastique, proche parente du cubisme. Eluard a donné à l'art respectif de Picasso les couleurs de l'angoisse et de la joie humaines.

L'amitié d'Apollinaire et de Picasso, toute la période qui va de 1905 jusqu'à la mort du poète, découpe dans l'œuvre du peintre une époque précise :

En un sens, le poète ne fait que se souvenir de lui-même à travers le souvenir émotif qu'il garde des caractères généraux les plus frappants des toiles de Picasso. Il regarde toujours les œuvres de ses amis peintres pour y retrouver son bien, le bien de la « poésie ». ${ }^{2}$

Il existe une sorte de circulation des valeurs, un type d'échange entre peinture et poésie. «La poésie peut parler la peinture, comme la peinture fixe pour les yeux la substance poétique: sentiments, fantaisie, imagination, goût de l'émerveillement et par-dessus tout, recherche du nouveau $»^{3}$.

Le poète d'Alcools a fait la connaissance de Picasso en 1903 et les deux hommes sont restés liés jusqu'à ce que la mort mît fin à leurs relations en 1918. Il a trouvé en lui la même tentation « orphique » qui inspirait toute sa poésie.

En effet, Apollinaire a toujours insisté sur la faculté maîtresse de Picasso : « sa puissance, sa capacité à chercher et à trouver » ${ }^{4}$ et sur sa prodigieuse faculté de « voir » le résumant en ces deux vers :

Ces yeux sont attentifs comme des fleurs qui veulent toujours contempler le soleil. ${ }^{5}$ 
C'est ainsi qu'il distingue le changement radical qui s'est produit dans l'œuvre du peintre depuis les deux périodes bleue et rose jusqu'au bouleversement « cubiste », la « création » a remplacé la « divination »:

Le calme vint après la frénésie. ${ }^{6}$

La poésie moderne, ce n'est pas Apollinaire. Avant lui, c'est Max Jacob ; après c'est Dada et André Breton. Un point commun les approche : une même attitude envers Picasso, faite de fascination et d'acceptation inconditionnelle. Max Jacob, pendant près de vingt ans, a été presque inséparable de l'artiste. Il disait à propos de son ami :

Je croyais en lui plus qu'en moi-même. ${ }^{7}$

C'est d'après les articles parus dans Europe que nous constatons qu'ils ont tout partagé entre eux: misère, génie et même logement. Max utilisait un ton particulier en parlant de Picasso comme «Le père Picasso », « Monsieur Picasso », « le génie : Pablo Picasso » et l'artiste a dit aussi beaucoup de bien sur le poète dans ses portraits. André Fermigier ${ }^{8}$ dit que «si l'on tient absolument à classer en «périodes» l'œuvre de Picasso, on devrait bien y trouver une «période Max Jacob ». » ${ }^{9}$

En laissant de côté les livres de critique tels que : Éluard, Picasso et la peinture de Jean-Charles Gateau, Et Picasso peint Guernica d'Alain Serres, Regards sur la peinture, Picasso de Roberto Manfrin, nous allons aborder la personne de Picasso d'après les revues parues entre 1925 et 1970.

Ce peintre a été un homme d'une curiosité et d'une impatience jamais « apaisées » vis-à-vis de l'art. Nous ne pouvons pas dire que Les Demoiselles d'Avignon ni ses tableaux de l'époque cubiste correspondent à l'art que le surréalisme propose. Il n’y a là ni «convulsion », ni « modèle purement intérieur », mais des variations sur des formes physiques dont la source est « cézannienne ». 
Si ses tableaux ressuscitent les visions de l'enfance, de la femme déformée, de la nature, qu'a-t-il de bien particulier pour nous occuper jusqu'à présent? Nous avons constaté que les peintres, les poètes et les journalistes ont traité chacun un des aspects de ce peintre. Selon les revues surréalistes comme La Révolution surréaliste, Le Surréalisme au service de la Révolution, les revues d'art comme Minotaure, les revues littéraires comme Europe, une nouvelle dimension accordée à ce grand peintre se fait sentir.

Ce sont des points de vue qui complètent ce que les livres tels Le Surréalisme et la peinture et L'image au service de la Révolution d'André Breton, Le Surréalisme de Michel Murat, Entretiens sur le Surréalisme de Ferdinand Alquié, Picasso de Christian Zervos, Picasso de Pierre Daix et Picasso le sage et le fou de Marie-Laure Bernadac, ont déjà souligné :

Un peintre, un homme, un génie. Un homme pour qui peindre veut dire voir, et qui mieux que personne "voit" son siècle. Visionnaire, oui. Engagé corps et biens dans son absolu. Homme public dont l'existence tumultueuse fascine les foules... mais aussi personnage farouche, secret, imprévisible. Un homme qui marque le $\mathrm{XX}^{\text {eme }}$ siècle d'une empreinte de feu. ${ }^{10}$

Pablo Picasso peut-être surréaliste quand il le veut, de la même manière qu'il peut être grec, baroque, réaliste ou nègre, sans jamais cesser d'être Picasso. Ami de Pierre Reverdy, de Guillaume Apollinaire, de Max Jacob, de Jean Cocteau, son affiliation au surréalisme, plutôt passive de sa part, est fonction de l'amitié qui le lia quelque temps à André Breton et, pendant toute sa vie, à Paul Eluard :

Il (Picasso) est devant un poème comme le poète devant un tableau. Il rêve, il imagine, il crée. Et voici que l'objet virtuel naît de l'objet réel, qu'il devient réel à son tour, voici qu'ils font image, du réel au réel, comme un mot avec tous les autres. ${ }^{11}$

Les revues sont peut-être le meilleur moyen auquel nous avons eu recours pour comprendre la dimension collective de Picasso. Elles ne sont pas seulement le 
lieu de publication préoriginale mais il faut les envisager comme des objets à part entière.

En analysant d'abord les revues surréalistes, André Breton publie le $4^{\text {ème }}$ numéro de la revue appelée d'abord La Révolution surréaliste puis Le Surréalisme au service de la Révolution, en juillet 1925, où il y fait de Picasso son $1^{\mathrm{er}}$ explorateur.

En 1933, Breton publie dans le premier numéro de Minotaure, un article intitulé Pablo Picasso dans son élément. Dans cet article, il mentionne que Picasso n'a jamais obéi qu'à sa propre pensée et que son système se découvre génial en présentant pour la première fois « un papillon naturel dans le champ d'un tableau, et qu'aussi il a pu le faire sans qu'aussitôt tout ce qui l'environne tombât en poussière, sans que les représentations bouleversantes que sa présence à cet endroit peut entraîner fissent en rien échec au système de représentations humaines dans lequel il est compris $»^{12}$.

Grâce à sa surprenante résolution, à sa plus mystérieuse et à sa plus sensible unité, il a fait passer l'inanimé à l'animé, la vie objective à la vie subjective. L'assimilation d'un organisme animal réel, par un mode de figuration brisant avec tous les modes conventionnels pourrait être, à elle seule, de nature à imposer silence à ses détracteurs :

Un sang fin, magnétique, se dépense généreusement d'un bord à l'autre de la ravissante cuve blanche, à peine plus grande qu'une main. $^{13}$

En effet, aux yeux de Picasso qui se préoccupe de pénétrer la couleur dans la sphère de la matière concrète, le bleu et le rouge ne sont conçus que comme états particuliers, presque négligeables dans leur particularité, de ce principe individualisant, concrétisant qu'est la couleur, unité de la lumière et de l'obscurité obtenue par l'intermédiaire de la transparence. Il confiait à Tériade ${ }^{14}$ :

Combien de fois au moment de mettre du bleu j'ai constaté que j'en manquais ! Alors j'ai pris du rouge et je l'ai mis à la place du bleu. ${ }^{15}$ 
Soulignons que la couverture de ce numéro est spécialement composée par Picasso : Minotaure assis avec un poignard. Il représente un homme tourné de trois quarts vers le spectateur, ayant la poitrine et les jambes gonflées, une courte queue et un énorme mufle de taureau et montrant un poignard à double tranchant.(Fig. 1)

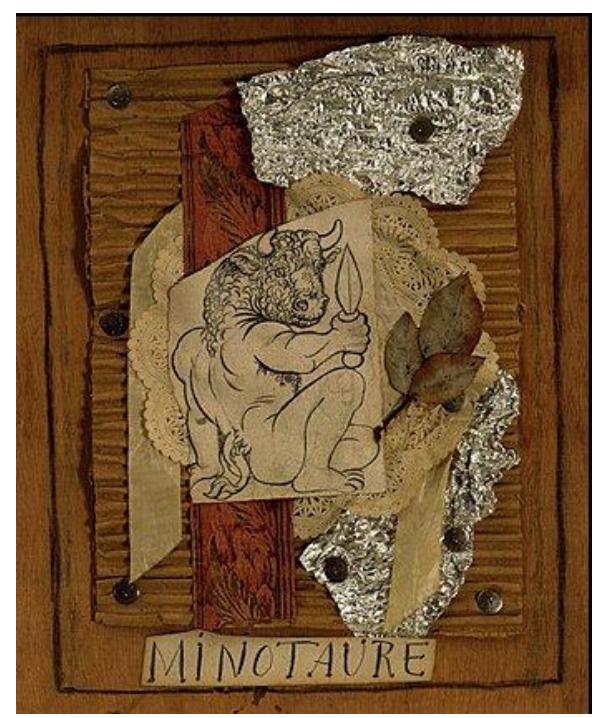

Figure 1. Picasso. Minotaure assis avec un poignard. 1933

Dans le cadre d'une enquête publiée dans Le Figaro Littéraire en 1955, Breton continue avec un décalage de trente ans et reprend par un article intitulé Réponse à une enquête, publié en 1961, il avoue que Picasso traite la femme «non comme sujet, mais comme objet, à la façon de la guitare ou de la sucette $»^{16}$. Selon ses règles, elle n'est qu'une structure physique dans l'espace «entraînée dans le même cycle de perturbation que le reste du mobilier ». L'écrivain souligne que seul Picasso a allumé « le feu d'artifice » dans sa prunelle lors des biais d'un numéro de Soirées de Paris, où Apollinaire offrait une reproduction «fumeuse » de cinq de ses natures mortes parues en 1913. Breton décrit ces toiles en disant que :

Quatre d'entre elles consistaient en un assemblage de matériaux de caractère résiduel tels que planchettes, bobines, chutes de linoléum, ficelle empruntés à la vie de tous les jours. Au choc provoqué par le jamais-vu succédait le sentiment d'un souverain équilibre de l'œuvre réalisée, promue à la vie organique et justifiant par là de sa nécessité. ${ }^{17}$ 
Selon ce grand poète, Picasso se distingue des peintres cubistes par «son lyrisme » qui lui a fait prendre de grandes libertés avec les données strictes que luimême et ses camarades s'étaient imposées :

Très vite chez lui, la rigide charpente du cubisme dit « analytique » est apparue secouée de grands vents et hantée. (...) La figuration s'est trouvée là bouleversée, révolutionnée de fond en comble. Opération titanesque à coup sûr. ${ }^{18}$

Ensuite, Picasso s'est tourné vers le surréalisme et «en témoignent une partie de sa production de 1923-1924, nombre d'œuvres de 1928 à 1930, les constructions métalliques de 1933, les poèmes semi-automatiques de 1935 et jusqu'au « Désir attrapé par la queue » de $1943 »^{19}$. Il s'attache au monde extérieur de «l'objet» et à la «cécité que cette disposition entretient sur le plan onirique et imaginatif $»^{20}$.

Parmi les nombreuses revues de cette période citons entre autres la Revue Europe. Depuis les années vingt, une revue comme Europe a pu se rendre orgueilleux de la fidélité de ses abonnés et de l'afflux régulier de lecteurs nouveaux, parce qu'ils y trouvaient une certaine chaleur de cœur qu'illustrent, entre autres, le nom de Picasso. De cela, les articles que nous avons trouvés dans quelques numéros sur l'artiste sont le témoignage émouvant.

Dans le $n^{\circ} 47$ de Décembre 1949, Paul Eluard écrit « une version intégrale du commentaire » pour un film d'après le célèbre tableau de Picasso Guernica :

Guernica. C'est une petite ville de Biscaye, capitale traditionnelle du Pays basque. (...) Guernica n'a qu'une importance historique et sentimentale. (...) Les avions allemands au service de Franco bombardèrent Guernica durant trois heures et demie par escadrilles se relevant tour à tour. La ville fut entièrement incendiée et rasée. Il y eut deux mille morts, tous civils. ${ }^{21}$

Dans le même numéro de la revue, Raymond Cogniat ${ }^{22}$ a donné un article intitulé Picasso commençant par ces mots : 
Plusieurs centaines de livres, plusieurs milliers d'articles n'ont pas épuisé le débat constamment ouvert et réouvert à propos de l'œuvre de Picasso et ce n'est pas sa dernière exposition à la Maison de la Pensée Française, ni ses poteries de Valauris, ni le Musée d'Antibes, et le succès que rencontrent ces diverses manifestations qui vont permettre de régler la question, bien au contraire. ${ }^{23}$

Pour l'auteur, l'art de Picasso est toujours comme une bombe qui « détonne dans le vide » et qui fait un bruit de fusée. Il a peut-être « un certain goût de la mystification qui explique en partie son art depuis plus de quarante ans, et qui ne diminue en rien son génie $»^{24}$. Il est toujours capable d'inventer de nouvelles formes et de nouveaux mondes. Personne plus que Picasso n'en a accumulé un plus grand nombre avec de constants changements et de constantes voltefaces. Mais une des caractéristiques de ce peintre est «de se refuser à soi-même l'exploitation de ses succès » et le public a accepté cet état de «révolte » et l'a transformé en une nouvelle convention, en un «académisme particulier» et en une "manière de routine ».

Picasso peut-il se tourner sans être victime de son propre succès ? se demande Cogniat :

Même le parti communiste français, qui prêche pour un art à base de réalisme social, pour l'expression la plus sentimentale des personnages et des thèmes, même le parti communiste que d'aucun croient désireux d'imposer une esthétique purement objective, même le parti communiste est très fier de compter parmi ses partisans un artiste comme Picasso, et ne peut lui refuser ses louanges, réussissant à trouver dans ses monstres, des caractères sensibles et humains ! $!^{25}$

Certes Picasso était l'homme du $\mathrm{XX}^{\text {ème }}$ siècle le plus révolté, le plus individualiste, le plus imité. Il a eu le plus d'influence sur l'esthétique de son temps et il a eu « le plus d'imitateurs ». Il a essayé d'échapper à lui-même en changeant de métier, il s'est réfugié dans la sculpture, dans la gravure, dans la céramique pour n'être pas enfermé dans sa peinture : 
Picasso-pompier rejoindrait le Picasso-révolutionnaire et serait bien capable par son génie indiscuté de faire accepter cette nouvelle métamorphose de soi-même. ${ }^{26}$

A la lecture de l'article de Raymond Cogniat, Pierre Abraham donne une Note sur l'article précédent dans le même numéro d'Europe. L'auteur a envoyé l'article de Cogniat à Jean Cassou pour prendre son avis et défendre Picasso :

Qui mieux que Jean Cassou, auteur de plusieurs études sur Picasso, conservateur en chef du Musée d'Art Moderne, membre de notre Comité et précédent directeur d'Europe, se trouvait qualifié pour donner dans le corps de la revue l'article destiné à équilibrer celui de Rayomnd Cogniat $?^{27}$

Mais en réponse à la lettre, Cassou mentionne que l'article de Cogniat ouvre des vues intéressantes sur « la psychologie de Picasso et le mécanisme de sa pensée créatrice $»^{28}$. Dès lors, Abraham a décidé de discuter lui-même l'article de Cogniat n’étant « ni un technicien de l'art moderne, ni un spécialiste de la peinture ».

Parmi tant de choses à relever, ce qui semble à Jean Cassou le plus « savoureux » dans l'article de Raymond, c'est qu'il donne à Picasso « une triple leçon de réalisme, d'expressionnisme et d'objectivité $»{ }^{29}$. Ce dernier cherche à créer une confusion entre le réalisme socialiste et le retour à un art académique ou « pompier». Mais Abraham regrette que l'article de Cogniat se glisse du plan esthétique au plan politique.

Personne n'est en fait «plus laborieux », «plus attentif aux règles du jeu » que Picasso comme l'affirme André Fermigier dans un extrait à propos d'une exposition à la Galerie Louise-Leiris paru dans Le Nouvel Observateur le 13 janvier 1969 :

Ses tableaux cubistes, Guernica, les natures mortes de 1924 nous le montrent capable d'une patience incroyable, exténuante, même si cette patience est moins didactique et programmée à la française que celle de Braques. ${ }^{30}$ 
Picasso est d'une haute conscience professionnelle, très accueillant, sachant et vivant la peinture au niveau d'une expérience parfois bouleversante et cruelle. La formule de Kahnweiler ${ }^{31}$ « son œuvre est passionnément autobiographique » ${ }^{32}$ montre son mystère changeant de style en changeant d'amour et n'ayant pas une grande familiarité avec son œuvre pour dater exactement chacun de ses tableaux :

1920 : c'est la paix, la vie facile, le «Train bleu », la redécouverte de la tradition et les débuts du musée imaginaire. 1937 : la guerre qui vient, le Paris de Sartre, la gloire de Freud, et des femmes impossibles qu'aucune psychanalyse ne parviendra à apaiser. 1945 : mère Méditerranée, des oursins, des maillots, des enfants, le Congrès de la paix et les remparts d'Antibes. ${ }^{33}$

Fermigier souligne dans son article que les rédacteurs de «Life » ${ }^{34}$ ont consacré à l'artiste un numéro spécial (Picasso and cubism, 1967) et qu'un critique anglais, John Berger, a écrit que le drame de Picasso est d'être « un anarchiste fou de soi-même, ni Français ni Espagnol, ni bourgeois ni prolétaire, complètement insensible à cette horizontalité bienfaisante et fourragère qu'assure l'engagement dans une classe, une époque, un destin collectif. » ${ }^{35}$

A un tout autre niveau, un autre critique anglais, Douglas Cooper, a dit que Picasso est « devant un dilemme dans ce sens qu'il s'est forgé un nouveau langage formel et une technique qui conviennent parfaitement à la représentation picturale de grands sujets à signification générale, alors que notre civilisation ne lui offre pas la possibilité de puiser l'inspiration dans les mythes communs ou des événements quotidiens. $)^{36}$

Après avoir donné quelques avis de critiques anglais sur Picasso, André juge et explique une série de quinze peintures numérotées et créées par Picasso en 1955, Les Femmes d'Alger, et d'une série de 58 peintures, Les Ménines, qu'il peint en 1957. Il dit que le peintre ne s'est pas intéressé aux Femmes d'Alger que «parce que le personnage de droite ressemblait comme un gant à la jeune femme qui venait alors d'entrer dans sa vie. Aux Ménines, parce que c'était un monde de poupées, 
d'enfants déguisés et parce que leur lumière était à peu près celle qu'il voyait dans son atelier de Cannes. $)^{37}$.

Bref, Fermigier dit que l'inspiration de Picasso vient des choses qui n'ont de signification que pour lui seul comme les «piqûres d'épingle », les souvenirs, les images, les spectacles, les personnages du cirque, les chapeaux des femmes, les personnages barbus, les moines, les gros hommes nus, les gitanes, les danseuses, l'Espagne plutôt «faite de débris picaresque, d'épaves de lectures et de vues de voyage $\gg{ }^{38}$.

Dans le n48.49 de l'année 1970 de la Revue Europe, nous trouvons plusieurs articles consacrés à Picasso. Premièrement l'article intitulé Picasso et les poètes, écrit par Charles Bachat et divisé en trois parties dont le premier, Apollinaire et l'orphisme de Picasso, souligne le fait qu'Apollinaire était toujours le commentateur dévoué et agressif des œuvres de Picasso depuis son premier article publié dans la Revue Immoraliste d'avril 1905 "Picasso, peintre et dessinateur » jusqu'à la préface au catalogue de l'exposition Matisse-Picasso à la galerie Paul Guillaume de février 1918.

Pour Pierre Reverdy, il n'est pas de contradiction entre les périodes qui ont précédé le « cubisme » et celles qui l'ont suivi car «Picasso a mené de front et avec la même incontestable maîtrise l'art qui prolonge pour lui l'ancienne tradition et celui qui pouvait être le fondement d'une tradition nouvelle $»{ }^{39}$.

D'après cette citation tirée de la seconde partie de l'article qui porte le titre d'Un aeil de lumière et de nuit, l'auteur met au clair Reverdy qui a toujours considéré l'aventure de Picasso comme une « compétition sportive ». Comme un tel champion de boxe, le peintre se montre l'athlète de la plus surprenante « envergure ». Il nie le pittoresque pour le dépouillement, il utilise la matière ou le concret pour les dépasser en créant un «lyrisme de la réalité » à mi chemin du réel et de l'image plastique ou poétique. 
Le génie multiforme de Picasso, dit Bachat dans sa $3^{\text {ème }}$ partie de l'article nommé Les frères voyants : Picasso et Eluard, a aussi touché les surréalistes. Ces derniers ont loué les collages de Picasso en les comparant à «un défi lancé à la peinture » pendant qu'ils défilaient eux-mêmes la poésie avec « les mots arrachés au quotidien, aux journaux, à la mode ». Tout en méprisant les objets luxueux, Picasso a regroupé dans plusieurs de ses toiles tout ce que la société a rejeté (Fig.2, 3) :

Il arriva que Picasso fit une chose très grave. Il prit une chemise sale et il la fixa sur une toile avec du fil et une aiguille. ${ }^{40}$

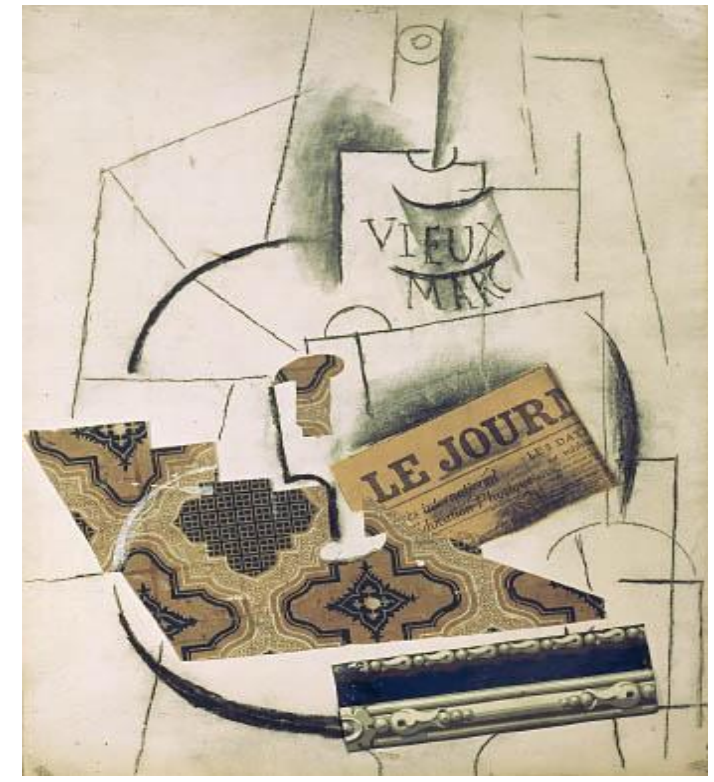

Figure 2. Picasso. Bouteille de Vieux-Mark, verre et journal (1913) 
Picasso à travers quelques périodiques entre 1925 et 1970

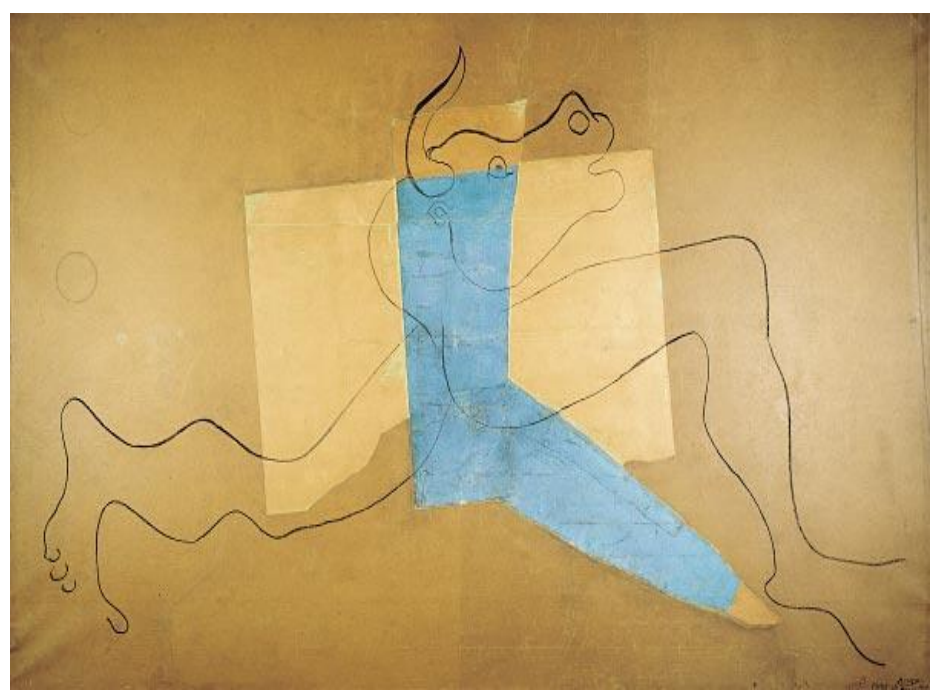

Figure 3. Picasso. Le Minotaure (1928)

Craie noire et papiers collés marouflés sur toile

Dans la Révolution Surréaliste de 1925, nous trouvons des reproductions des Demoiselles d'Avignon et des Trois danseurs (Fig.4) que Pierre Daix commente ainsi :

Picasso introduit dans sa toile un autre élément caractéristique de la nouvelle école, élément aussi bien emprunté au réel que le jazz, les éclairages électroniques colorés des plateaux et des boîtes de nuit. ${ }^{41}$ 


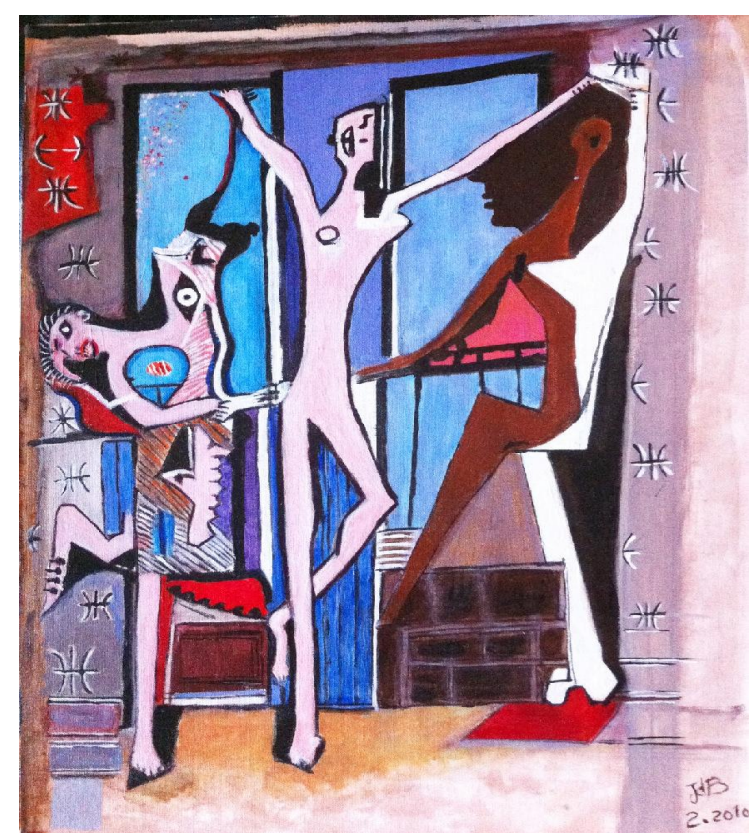

Figure 4. Picasso. Les Trois Danseurs (1923)

Cependant, Picasso a fait la connaissance d'Eluard depuis cette grande époque surréaliste. Dès ce moment, nous trouvons que la plupart des recueils du poète sont accompagnés d'un «commentaire plastique » de son ami sous une forme de «dessins, d'huiles ou de lithographies et de signes aquarelles » comme pour Le Livre ouvert (Fig 5). L'influence du peintre donna à la poésie éluardienne une sorte d'ouverture vers la réalité sociale, un passage de «l'horizon d'un seul à l'horizon de tous ».

Charles Bachat conclut son article en appliquant à Picasso la formule de Cocteau :

Lorsqu'une œuvre semble en avance sur son époque, c'est simplement que son époque est en retard sur elle. ${ }^{42}$

C'est dans ce même numéro de la revue que nous trouvons un article d'Hélène Henry intitulé Max Jacob et Picasso (Jalons Chronologiques pour une amitié : 1901-1944) où l'auteure montre les étapes et l'ascension de l'amitié 
Picasso/Max Jacob année par année. En 1901, c'était la première exposition de Picasso chez Ambroise Vollard, rue Laffitte, à Paris. Vaguement critique d'art, Max Jacob s'est rendu, admirant et griffonnant son enthousiasme sur une carte de visite et le lendemain a été peint le premier portrait du poète par Picasso.

C'est également en retournant à Barcelone, au début de 1903, que Picasso dessine un conte L'histoire simple et claire de Max Jacob dans lequel nous voyons le poète à sa table de travail «écrivant d'abondance». «Le haut-de-forme des grands jours jeté par terre. Puis, chapeauté cette fois, il se rend chez un éditeur, lui lit ses manuscrits, en sort des billets de banque plein les mains en criant : «Olé ! »» ${ }^{43}$ (Fig.5).

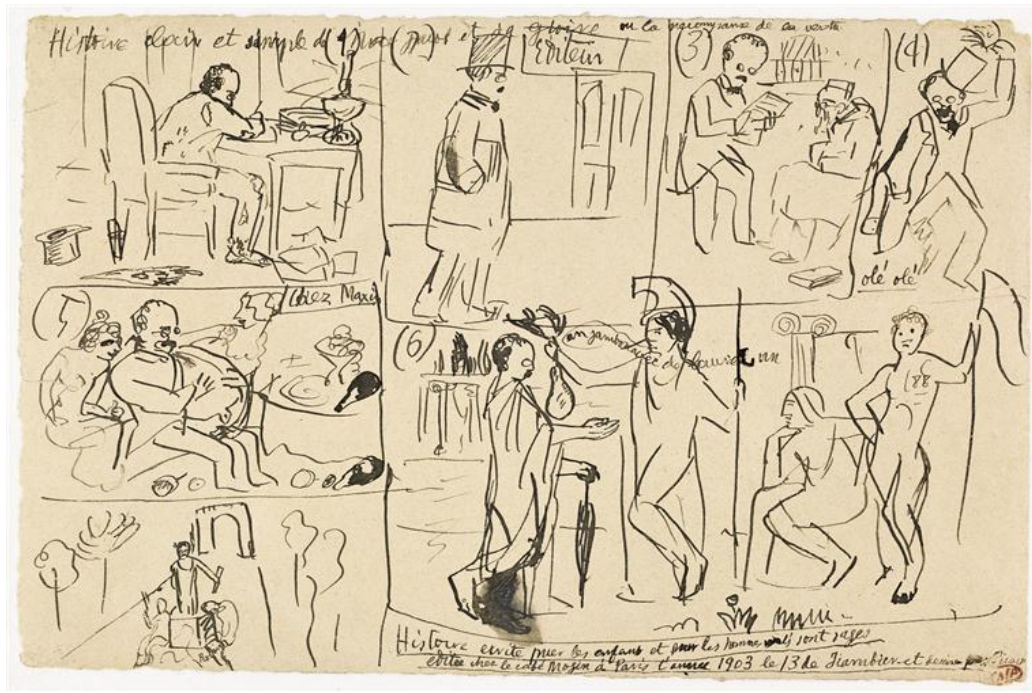

Figure 5 : Picasso. Histoire simple et claire de Max Jacob (1903)

En avril 1904, Picasso repartit pour Paris, « voyage sans retour » et s'installa dans un atelier de la « Maison du Trappeur » bientôt rebaptisée « bateaulavoir ». Mais pour négocier les toiles et les dessins de Picasso, Max se trouvait toujours boulevard Barbès. 
L'année d'après, 1905, dans La Plume, revue littéraire et artistique française, paraît un article intitulé Les jeunes : Picasso peintre, écrit par Guillaume Apollinaire et publié en 1905. Là Hélène Henry a mentionné que Michel Ange admire surtout les qualités de la bonne peinture qui se trouvent dans les tableaux de Pablo Picasso : «le discernement», «l'aisance », « la substance » et «le nerf». André Salmon de même, contrairement à ses habitudes, a donné un poème au numéro un et unique de la revue Immoraliste fondée par Apollinaire. C'est Le Cheval (2), dédié à Picasso, «Le Cheval à Picasso dont ne se laissait pas notre bande... $\rangle^{44}$. Apollinaire par ailleurs continue à porter à Picasso une admiration vigoureuse. Un Calligramme Pablo Picasso paraît dans le numéro 17 de Sic.

Depuis le début de 1907, Picasso a commencé à dessiner sa très grande œuvre Les Demoiselles d'Avignon dont le titre fait allusion à la rue Avignon de Barcelone, une rue du quartier des prostituées. D’où cette légende de «la grand'mère de Max » qui a été l'une des femmes de ce tableau.

Quand Picasso n'écrit pas à son ami Max lors de son voyage chez ses parents, il écrit à Kahnweiler, nous dit Hélène, pour lui demander «pourquoi Picasso ne m'écrit-il pas ? Est-ce qu'il est fâché ? » de telle sorte que ces questions reviennent « comme un refrain ». Alors qu'à la fin de l'année 1914, Picasso a fait de Max Jacob un portrait magnifique: «c'est un dessin à la mine de plomb qui représente Max assis, de face, avec un sourire un peu énigmatique ${ }^{45}$ (Fig 6). 
Picasso à travers quelques périodiques entre 1925 et 1970

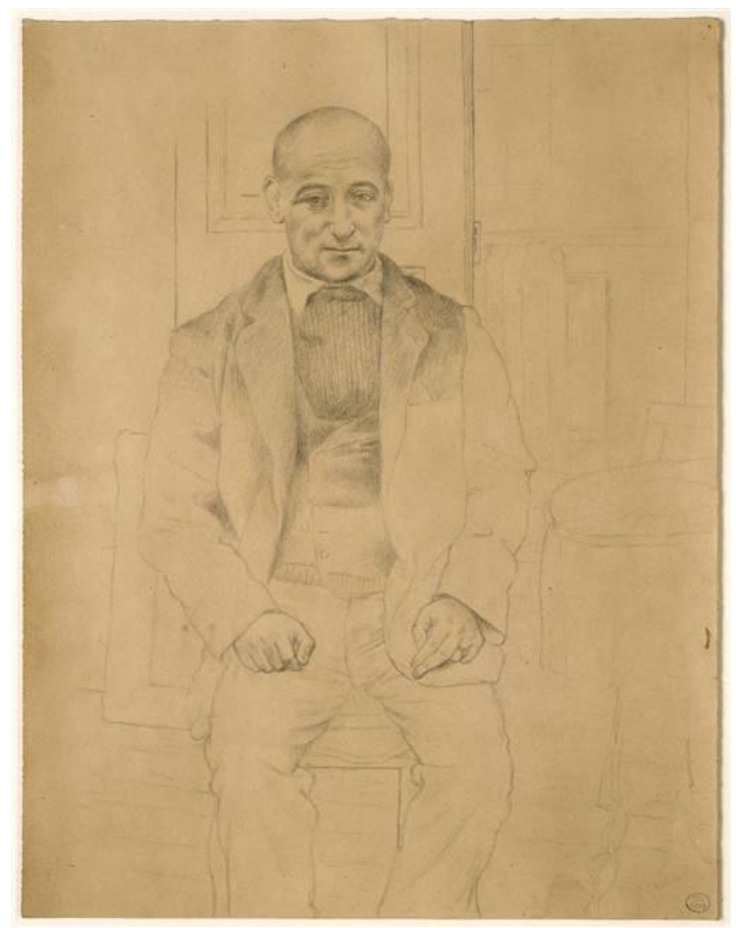

Figure 6. Pablo Picasso. Max Jacob (1915)

A son tour, Max publie en 1918 Le Phanérogame écrit en 1905, «c'est l'histoire d'un homme qui volait par agitation des cuisses ». L'édition originale comporte une gravure de Picasso: «Picasso figure d'ailleurs d'une manière inattendue dans ce livre dédié à André Salmon » ${ }^{46}$ (Fig.7).

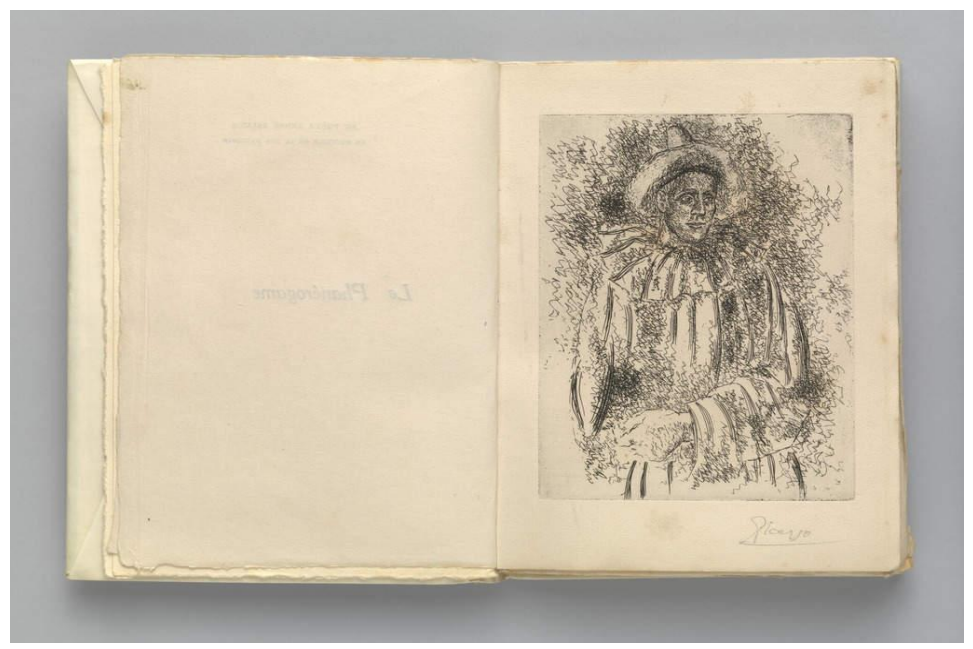


Figure 7. Picasso. Le Phanérogame (1918)

C'est en 1919 que le poète publie La Défense de Tartufe, avec une eau-forte de Picasso. Dans Le Laboratoire Central qui paraît en 1921, Jacob donne l'un de ses plus beaux poèmes, Honneur de la Sardane et de la Tenora, qui a été écrit dès 1913 pour Picasso. En 1927, aux Cahiers d'Art de Christan Zervos, Max publie aussi un long texte de souvenirs sur le peintre et en 1943, écrivant à Marcel Béalu ${ }^{47}$ lui disant :

Ce qui explique une amitié de quarante et un années avec Picasso c'est que nous avions passé un pacte : Tout ce qu'on te dira de moi ne comptera pas et réciproquement. ${ }^{48}$

Dans le même numéro de cette Revue d'Europe, Raymond Jean donne un article publié sous le titre d'Eluard et Picasso. L'amitié de ces deux personnages, dès l'époque surréaliste, se place sous le signe de la "générosité», de la « tendresse » et de « l'intelligence lucide ».

D’une quinzaine d'année plus jeune que le peintre, Eluard a trouvé en Picasso « quelque chose qui le fascine, qui l'inspire, le guide, lui apprend à mieux voir et lire la réalité. ${ }^{49}$

Bref, il est l'introducteur à la réalité par excellence.

Qui a pu être le plus proche de Picasso ? Eluard ?! Et qui a plus intelligemment parlé de lui ? Qui a mieux dit, mieux saisi, mieux fixé, en peu de mots, les gestes de quelques-uns des plus grands peintres d'aujourd'hui ? Eluard loue ce lyrisme et ce réalisme qui lui sont propres :

Homme, femme, statue, table, guitare, redeviennent des hommes, des femmes, des statues, des tables, des guitares, plus familiers qu'auparavant, parce que compréhensibles, sensibles à l'esprit comme aux sens. 50 
C'est dans ce même numéro d'Europe que Michel Pierssens, professeur titulaire à l'université de Montréal, que j'ai eu l'honneur de rencontrer, a publié un article intitulé Apollinaire, Picasso, et la mort de la poésie. Dans cet article, il démontre la forte relation entre Apollinaire et Picasso rapportant que le poète a fondé en 1905 La Revue Immoraliste d'un unique numéro «pour inaugurer la longue série de ses futurs articles sur Picasso » :

On a dit de Picasso que ses œuvres témoignaient d'un désenchantement précoce. Je pense le contraire. Tout l'enchante et son talent incontestable me paraît au service d'une fantaisie qui mêle justement le délicieux et l'horrible, l'abject et le délicat. ${ }^{51}$

Jusqu'aux premières réalisations du Cubisme, jusqu'à « la période nègre », déclare Pierssens, les toiles de Picasso parlaient de la sensibilité et non de la subjectivité. C'est pourquoi Apollinaire le considère comme un nouveau Prométhée ${ }^{52}$ reconduit à la divinité et dont la main est dirigée par un être inconnu qui se sert d'eux «comme un instrument». Pour lui, tout doit mêler le monde de l'art au monde moral, « un univers où les vertus sont plastiques, où la peinture est pureté, unité, vérité » ${ }^{53}$. De là, « on s'achemine vers la peinture pure ». Rappelons ces vers d'Apollinaire dans le drame surréaliste Mamelles de Tirésias :

Le fils :

On apprend de Montrouge

Que Monsieur Picasso

Fait un tableau qui bouge

Ainsi que ce berceau

Le Mari :

Et vive le pinceau

De l'ami Picasso ${ }^{54}$

Apollinaire n'a jamais décrit les toiles de Picasso comme il a fait avec celles de Delaunay. Cependant, il a parlé toujours du peintre en tant qu'homme ou plutôt en tant que surhomme. Pour lui, Picasso a rejoint « la galerie des génies » :

Picasso est l'hoir de tous les grands artistes... 55

Dans les Calligrammes, le poète, inspiré de la peinture, essaye à la réalisation de l'objectif idéaliste, à la synthèse des arts. Ainsi que Michel Décaudin ${ }^{56}$ 
le précise «l'introduction de lettres ou de mots dans leurs tableaux, que les peintres cubistes pratiquaient à partir de 1911, surtout les compositions futuristes, à caractère idéogrammatique, étaient pour lui une incitation au dépassement des oppositions entre les arts. Ce que les symbolistes avaient attendu de la musique, il le demande au dessin et à la peinture. $»^{57}$

Pour conclure : disons que aimer ou ne pas aimer Picasso, personne ne peut nier la qualité et la diversité de ses moyens d'expression. Dans la majorité des revues anglaises, américaines, italiennes ou espagnoles figurent une nouvelle figure, une nouvelle analyse, un nouveau commentaire concernant Picasso. Dans Vanity Fair c'est une nouvelle présentation de ce grand peintre, une grande liste d'articles peuvent être abordés dans le but d'immortaliser ce grand talent qu'est Picasso. 


\section{$\underline{\text { Notes }}$}

1 BACHAT (Charles) : "Picasso et les poètes » Europe N48.49 (Avril 1, 1970), Editions Reider, Paris, p.165

2 PIERSSENS (Michel): “Apollinaire, Picasso, Et la mort de la poésie » Europe, N48.49 (Avril 1, 1970), Op.cit, p.180

${ }^{3}$ lbid, p.182

${ }^{4}$ BACHAT (Charles) : « Picasso et les poètes » Europe N48.49 (Avril 1, 1970), op.cit, p.166

${ }^{5}$ Ibid, p.167

${ }^{6}$ Ibid.

${ }^{7}$ PIERSSENS (Michel): “Apollinaire, Picasso, Et la mort de la poésie » Europe, N48.49 (Avril 1, 1970), Op.cit, p.189

${ }^{8}$ FERMIGIER (André) était un historien d'art, critique et éditeur français de la seconde moitié du XX ${ }^{\text {ème }}$ siècle.

${ }^{9}$ HENRY (Hélène) : "Max Jacob et Picasso » Europe. N48.49, (Avril 1, 1970), op.cit, p.193

${ }^{10}$ BERNADAC (Marie-Laure) : Picasso le sage et le fou, Editions Gallimard, Paris, 1986, 4 ème de couverture.

${ }^{11}$ JEAN (Raymond) : « Eluard et Picasso ». Europe N48.49 (Avril 1, 1970), op.cit, p.39

12 BRETON (André): "Picasso dans son élément " Minotaure. Numéro 1, Editions Skira, Paris, 1933, p. 10

${ }^{13}$ Ibid.

${ }^{14}$ Tériade était un critique d'art et un éditeur français, d'origine grecque, d'ouvrages d'art.

${ }^{15}$ Ibid.

16 BRETON (André) : "Réponse à une enquête ». Le Surréalisme et la peinture, Editions Gallimard, Paris, 1965, p.115

${ }^{17}$ Ibid. p.116

${ }^{18}$ Ibid. p.117

${ }^{19}$ Ibid.

${ }^{20}$ Ibid.

${ }^{21}$ ELUARD (Paul) : « Guernica » Europe. N47 (Décembre 1949), Editions Reider, Paris, p.47

${ }^{22}$ Critique d'art réputé et rédacteur en chef hebdomadaire consacré particulièrement aux arts plastiques.

${ }^{23}$ COGNIAT (Raymond) : « Picasso » Europe. N47 (Décembre 1949), op.cit, p.51

${ }^{24}$ Ibid, p.52

${ }^{25}$ Ibid, p.53

${ }^{26}$ Ibid, p.54

${ }^{27}$ ABRAHAM (Pierre) : "Note sur l'article précédent ». Europe. №47 (Décembre 1949), op.cit, p.54

${ }^{28}$ Ibid, p.55

${ }^{29}$ Ibid, p.56

${ }^{30}$ GEERINCK (Marianne) : "Le Nouvel Observateur » Histoire de l'art, le XX ${ }^{\text {ème }}$ siècle : du Fauvisme au Surréalisme - une anthologie, Editions Institut supérieur d'architecture Victor Horta, Bruxelles, 1989-1990, p.58

${ }^{31}$ KANHWEILER (Daniel-Henry) est un écrivain, collectionneur et marchand d'art Allemand.

${ }^{32}$ Ibid.

${ }^{33}$ Ibid. 
${ }^{34}$ Life est un magazine américain hebdomadaire de 1883 à 1972.

${ }^{35} \mathrm{lbid}$.

${ }^{36}$ Ibid, p.59

${ }^{37}$ Ibid.

${ }^{38}$ Ibid.

${ }^{39}$ BACHAT (Charles) : « Picasso et les poètes » Europe N48.49 (Avril 1, 1970), op.cit, p.171

${ }^{40}$ Ibid, p.173

${ }^{41}$ Ibid, p.174

42 Ibid, p.177

${ }^{43}$ HENRY (Hélène) : « Max Jacob et Picasso ». Europe N48.49 (Avril 1, 1970), op.cit, p.194

${ }^{44}$ Ibid, p.196

${ }^{45}$ Ibid, p.204

${ }^{46}$ Ibid, p.206

${ }^{47}$ BEALU (Marcel) est un écrivain et un poète français du XXème siècle.

${ }^{48}$ Ibid, p.209

${ }^{49}$ JEAN (Raymond) : « Eluard et Picasso ». Europe N48.49 (Avril 1, 1970), op.cit, p.37

${ }^{50} \mathrm{Ibid}$.

${ }^{51}$ PIERSSENS (Michel): “Apollinaire, Picasso, Et la mort de la poésie » Europe, N48.49 (Avril

1, 1970), Op.cit, p.179

${ }^{52}$ Dans la mythologie grecque, Prométhée est un Titan (une divinité primordiale géante).

53 Ibid, p.184

${ }^{54}$ Ibid, p.185

${ }^{55}$ Ibid, p.187

${ }^{56}$ DÉCAUDIN (Michel) était un professeur de lettres français.

${ }^{57}$ Ibid, p.188

\section{Bibliographie}

\section{Corpus :}

ABRAHAM (Pierre) : «Note sur l'article précédent ». Europe, №47 (Décembre 1949), Editions Reider, Paris, p.54-59.

BACHAT (Charles) : «Picasso et les poètes » Europe, $N^{\circ} 48.49$ (Avril 1, 1970), Editions Reider, Paris, p.165-177

BRETON (André): «Picasso dans son élément » Minotaure, Numéro 1, Editions Skira, Paris, 1933, p.10-22.

BRETON (André) : «Réponse à une enquête ». Le Surréalisme et la peinture, Editions Gallimard, Paris, 1965, p.153-158. 
Picasso à travers quelques périodiques entre 1925 et 1970

COGNIAT (Raymond) : «Picasso » Europe, ํ47 (Décembre 1949), Editions Reider, Paris, p.51-54

ELUARD (Paul) : «Guernica » Europe, N47 (Décembre 1949), Editions Reider, Paris, p. $47-50$

GEERINCK (Marianne) : «Le Nouvel Observateur » Histoire de l'art, le XX $X^{\text {me }}$ siècle : du Fauvisme au Surréalisme - une anthologie, Editions Institut supérieur d'architecture Victor Horta, Bruxelles, 1989-1990, p.58-60

HENRY (Hélène) : «Max Jacob et Picasso ». Europe, N48.49 (Avril 1, 1970), Editions Reider, Paris, p.191-210

JEAN (Raymond) : «Eluard et Picasso ». Europe, №48.49 (Avril 1, 1970), Editions Reider, Paris, p.37-41

PIERSSENS (Michel): “Apollinaire, Picasso, Et la mort de la poésie » Europe, $\mathrm{N}^{\circ} 48.49$ (Avril 1, 1970), Editions Reider, Paris, p.178-190

\section{Ouvrages consacrés à Picasso :}

GATEAU (Jean-Charles) : Eluard, Picasso et la peinture, Editions Librairie Droz S.A, Genève, 1983, 337p.

DAIX (Pierre): Tout l'œuvre peint de Picasso, périodes bleue et rose, Editions Flammarion, Paris, 1980, 119p.

DAIX (Pierre): Picasso, Editions Aimery Somogy s.a, Paris, 1982. 128p.

DAIX (Pierre): Les après guerre de Picasso (1945-1955) et sa rupture avec Aragon, Editions Ides et Calendes, Italie, 2006, 180p.

\section{Dictionnaires :}

DAIX (Pierre) : Le Nouveau dictionnaire Picasso, Editions Robert Laffont, Paris, 2012, 950p. 